\title{
Event-related Potentials-based Evaluation of Attention Allocation while Watching Virtual Reality
}

\author{
Ryo Ogawa, Kaito Kageyama, ${ }^{*}$ Yasushi Nakatani, ${ }^{* *}$ Yumie Ono, ${ }^{* * *}$ Shingo Murakami ${ }^{*}$ \#
}

\begin{abstract}
Virtual reality (VR) has been applied to several fields such as entertainment, education, and medicine in recent years. VR is characterized by a high sense of immersion, which can be represented by the attention allocation from the real world to the virtual space. Although a high degree of attention allocation is significant in VR technology, most existing evaluation methods of VR applications are based on subjective questionnaires. Thus, quantitative and objective VR application evaluation methods are needed to realize advanced VR applications. In this study, we adopted a probe stimulus method to evaluate the attention allocation quantitatively and objectively in VR technology. Ten young adult participants underwent an auditory oddball task while they experienced VR content. The amount of attention directed to the VR content could be quantified based on the decrease in the event-related P300 wave response in the case of the oddball task. The participants watched two-dimensional and three-dimensional VR contents on a liquid crystal display and a headmounted display, respectively, while brain activity was recorded in the form of electroencephalographic signals. A total of 230 probe stimuli at $1800 \mathrm{~Hz}$ (standard stimulus), $2000 \mathrm{~Hz}$ (target stimulus), and $500 \mathrm{~Hz}$ (deviant stimulus) were presented randomly via an earphone for $70 \mathrm{~ms}$ at $1000-\mathrm{ms}$ intervals at the fractions of 70 , 15 , and $15 \%$, respectively. Additionally, the reaction time and false reaction rate during the oddball task were measured as behavioral measures, and a questionnaire was used for subjective evaluation after the task. Based on a comparison of the subjective measure, behavioral measure, and amplitudes of P300 measured with the target stimulus from $\mathrm{Pz}$ and deviant stimulus from $\mathrm{Cz}$, we found that attention allocation to the VR content can be quantitatively estimated using the amplitude of P300 for the deviant stimulus. These results suggest that the proposed method involving event-related potentials can be used as an indicator for attention allocation while watching VR content.
\end{abstract}

Keywords: VR, ERP, P300, oddball task, probe stimulus method.

Adv Biomed Eng. 11: pp. 1-9, 2022.

\footnotetext{
This study was presented at the Symposium on Biomedical Engineering 2021, September, 2021.

Received on August 3, 2021; revised on October 31, 2021; accepted on November 30, 2021.

* Department of Electrical, Electronics, and Communication Engineering, Faculty of Science and Engineering, Chuo University, Tokyo, Japan.

** Faculty of Economics, Chuo University, Tokyo, Japan.

**** Department of Electronics and Bioinformatics, School of Science and Technology, Meiji University, Kanagawa, Japan.

\# 1-13-27 Kasuga, Bunkyo-ku, Tokyo 112-8551, Japan.

E-mail: murakami@elect.chuo-u.ac.jp
}

\section{(cc) BY}

Copyright: (02022 The Author(s). This is an open access article distributed under the terms of the Creative Commons BY 4.0 International (Attribution) License (https:// creativecommons.org/licenses/by/4.0/legalcode), which permits the unrestricted distribution, reproduction and use of the article provided the original source and authors are credited.

\section{Introduction}

Since its introduction in the late 1980 s, virtual reality (VR) technology has been applied to a broad range of fields such as entertainment, education, and medicine [1]. In recent years, the successful commercialization of stand-alone, head-mounted displays (HMDs) has been realized by the increasing processing power of computers [2], which allows high-definition and threedimensional (3D) images which are excellent resemblances of reality to be rendered with a wide viewing angle by providing parallax to both eyes, without extra information from the real world or awareness of the presence of the HMD. This creates striking reality in VR devices and a very immersive visual experience. However, since full-fledged VR has been realized in recent years, a consensus definition and underlying mechanism of VR immersion have not been established. It is not clear what factors contribute to the sense of immersion. A reason for 
this is that immersion had been evaluated only subjectively. Therefore, an objective and quantitative evaluation method is required to realize enhanced VR content.

To study VR immersion in the present study, we assumed that VR immersion is the separation from the real world and the attention allocated from the real world to the virtual space, and that it can be explained by a decrease in attention allocated to the real world and an increase in attention allocated to the to the virtual space. In the present study, the evaluation method for attention allocation in P300 and oddball task used in previous studies was adopted to evaluate attention allocation [3-5]. The P300, which is an event-related potential (ERP) component that appears as a positive peak at approximately $300 \mathrm{~ms}$ after a decision-making event, is obtained using an oddball task, and it has been employed for quantitative and objective evaluation of task workload [3-5]. The oddball paradigm is an experimental procedure wherein two or more types of stimulus events are presented at different frequencies of appearance. The stimuli that appear less frequently and the subjects respond to are called target stimuli (e.g. high tone), whereas the stimuli that appear most frequently and the subjects do not respond to are called standard stimuli (e.g. normal tone). The stimuli that appears less frequently and the subjects do not respond to are called deviant stimuli (e.g. low tone). It has been demonstrated that the P300 dominant in the central region of the brain appears with short latencies for deviant stimuli, and the P300 dominant in the parietal region appears with long latencies for target stimuli [6-8]. The probe stimulus method is used to estimate the amount of attention directed to a task or situation from the response to a stimulus indirectly, by leveraging the fact that the amplitude of P300 increases when focusing on stimuli, and decreases otherwise. When the amount of attentional resources is assumed to be constant, the ratio of the amount of attention directed to the image and probe stimulus changes in high- and low-interest images, and the amplitude of P300 for the high-interest image becomes smaller than that of the low-interest image. We utilized this property of the probe stimulus method to evaluate the attention allocation objectively and quantitatively while watching VR by replacing the image with VR contents so that the attention allocation while watching the VR video can be evaluated.

In this study, we focused on the visual part of VR, and examined the sense of immersion from the viewpoint of attention by using the probe stimulation method in the oddball task. Previous studies examined the difference in the extent of attention according to the genre of video and the difference in the extent of attention between videos with and without sound $[9,10]$. However, the amount of attention required when viewing the 3D video using HMD was not examined. We examined the amount of attention toward a 3D video using an HMD and a two-dimensional (2D) video and a still image using a liquid crystal display (LCD) evaluated with subjective, behavioral, and physiological measures. Subjective and behavioral measures were collected by measuring subjective assessments, reaction times, and false response rates. The results suggest that ERPs are a useful indicator of VR immersion. Furthermore, considering that viewing of $3 \mathrm{D}$ videos using HMDs will become more widespread in the future, the quantitative evaluation of the sense of immersion in this study may serve as an index to realize a more immersive $3 \mathrm{D}$ video content.

\section{Methods}

\subsection{Subjects}

Ten healthy adult males [aged $22.5 \pm 1.3$ years (mean \pm standard deviation)] participated in the experiment. The subjects did not have any neurological and psychiatric disorders and had no experience with HMDs. The experiment was approved by Chuo University Ethics Review Committee. Informed consent was obtained from all participants.

\subsection{Visual stimulus}

An HMD (Oculus Quest 2, viewing angle 100 degree; resolution $1832 \times 1920$ ) was used to present the 3D video. The video to be shown to the participants in the experiment was approximately $6 \mathrm{~min}$ in length, which is suitable for additive averaging with only negligible viewpoint movement and body engagement. In the experiment, we used a VR video called "Land of Starry Skies," which was shot near Lake Tekapo in South Island, New Zealand. The 3D video was presented using the HMD, and the corresponding 2D version and a still image from one scene of the 2D video were visualized with an LCD (width 15.6 inch, resolution $1920 \times 1080$, viewing distance: $50 \mathrm{~cm}$ ) of a laptop computer (HP OMEN).

\subsection{Auditory stimulus}

As probe stimuli, sine waves of $1800 \mathrm{~Hz}$ (standard stimulus), $2000 \mathrm{~Hz}$ (target stimulus), and $500 \mathrm{~Hz}$ (deviant stimulus) were presented in random order from an earphone for durations of $70 \mathrm{~ms}$ at fractions of 70,15 , and $15 \%$, respectively. The interval between stimuli was $1000 \mathrm{~ms}$, and the total number of stimuli presented was 230.

\subsection{Experimental procedure}

The participants were instructed to press the space bar key on the keyboard of their respective PCs with their 
dominant hand in response to the target sound while they watched videos or still image. A subject was seated in a chair with his dominant hand touching the space key of the laptop computer. The subject was asked to assume a comfortable sitting position. In order to prevent noise generation, the subject was instructed to avoid movement of the head as much as possible. The subject was also instructed to minimize blinking, eye movements, and any body movements associated with pressing the space key. A three-tone oddball task was presented using a stimulus presentation software (Psychopy), and the timing of the stimulus was synchronized by inputting a trigger to an EEG (PolymateV AP5148, Miyuki Giken). The videos and image were presented to the participants in random order. A 5-min break was permitted between measurements. During the break, a questionnaire of subjective evaluation [10] was presented to the participants. The videos and image were shown for $6 \mathrm{~min}$, and the sound stimulus was started $15 \mathrm{~s}$ after the onset of the video.

\subsection{Subjective measures}

After the participants viewed each block of the video, a questionnaire on the subjective measure (Figs. 1-3) was presented to them. Based on previous studies to evaluate the attention allocation while watching $2 \mathrm{D}$ video $[9,10]$, subjective ratings were obtained by using a 9-point scale for the extent of attention directed to the sound (1: low, 9: high) and video (1: low, 9: high), and the interestingness of the video (1: boring, 9: interesting). They mean ratings were analyzed by paired $t$-test with Bonferroni correction. $P$-values less than 0.01 or 0.05 are shown above the bar chart, while $p$-values greater than 0.05 are not shown in the figure. The extent of attention directed to the sound was quantified to evaluate the subjective measure of the amount of attention directed to the auditory oddball task. Previous studies reported that more attention was directed to the video, and less attention to the sound $[9,10]$. The scores for the amount of attention directed to the sound and to the video were summed to examine if the difference in visual stimulus method and resulting difference in interestingness affect the constant attention resource that is assumed in the probe method.

\subsection{Behavioral measures}

As behavioral measures, we measured the reaction time from the presentation of the target stimulus to the button press, the failure rate for the target stimulus, and the false alarm (FA) rate for the standard and deviant stimuli. The results are presented as mean with standard deviation (SD), and $\mathrm{p}$ values obtained from paired $t$-test with Bonferroni correction are shown only when $p<0.01$ or 0.05 (Figs. 4-6). $P$-values greater than 0.05 are not shown in the figures. The failure rate was evaluated as the percentage of trials with no response to the target stimulus that was presented (Fig. 5). The FA rate was evaluated as the percentage of incorrectly responded trials among the standard and deviant stimulus presentations (Fig. 6).

\subsection{EEG signal recording}

The EEG signals were recorded at two locations $(\mathrm{Cz}$ and $\mathrm{Pz}$ ) on the midline. The left earlobe (A1) was used as the reference electrode and the right earlobe (A2) for the ground. The electrooculogram (EOG) signals were also recorded from the right eye to monitor eye movement and blinks. The impedance of the electrodes at all sites was maintained at $10 \mathrm{k} \Omega$, and the signals were recorded using a band frequency of $0.05-20 \mathrm{~Hz}$ and a sampling frequency of $200 \mathrm{~Hz}$.

\subsection{Data analysis}

The measured EEG signals were analyzed using EEGLAB and ERPLAB on MATLAB. Trials containing fluctuations greater than $\pm 100 \mu \mathrm{V}$ within an analysis segment in the EEG and EOG channels were automatically excluded. In the remaining trials, those containing artifacts by manual inspection were excluded. Trials obtained from three participants contained large fluctuations only for the target stimulus following pressing of the space bar. These were excluded from the analysis for P300. The EEG waveforms from $200 \mathrm{~ms}$ before stimulus presentation to $800 \mathrm{~ms}$ after stimulus presentation were additively averaged for each participant, electrode, visual stimulus type, and stimulus type separately to obtain the peak amplitude and latency at the dominant region (target stimulus: Pz, deviation stimulus: $\mathrm{Cz}$ ). The maximum positive peak that occurred between $250 \mathrm{~ms}$ and $600 \mathrm{~ms}$ after the stimulus presentation was identified as the P300 amplitude. The amplitudes of the identified $\mathrm{P} 300$ are presented as mean and $\mathrm{SD}$, and $p$ values $<0.01$ or $<0.05$ obtained from paired $t$-test with Bonferroni correction are shown above the bar chart (Figs. 8-12). $P$ values greater than 0.05 are not shown in the figure. Because the difference in magnitude of P300 amplitudes among the participates was much larger than that among the three stimulus methods, the correlation between components was masked by the difference among participants and the effects of the difference among the three stimulus methods could not be represented by correlation coefficient. Furthermore, the total ERP waveform was calculated by averaging the individual ERP waveforms obtained from all participants in the experiments (Figs. 7 and 10). 


\section{Results}

\subsection{Subjective measures}

\subsubsection{Attention to auditory stimulus}

The ratings of the subjective evaluation of the amount of attention directed to the sound while watching 2D still image, and 2D and 3D videos $(n=10)$ were averaged, and are shown in Fig. 1 with error bars denoting the SD.

As expected, the amount of attention directed toward sound was the highest for the still image, followed by $2 \mathrm{D}$ video and $3 \mathrm{D}$ video in that order. However, no significant differences were found between groups $(p>$ 0.05 for all pairs). These results suggest that subjective evaluation of attention to auditory stimulus is not associated with the different visual stimuli and cannot be used to measure the attention allocation in this case.

\subsubsection{Attention to visual stimuli}

Subsequently, we evaluated the differences in attention directed to the image and videos while watching 2D still image, and 2D and 3D videos (Fig. 2).

The subjective rating of attention directed to images was the highest in 3D video, followed by 2D video and still image (in that order), contrary to the amount of attention directed to the sounds. However, the differences were more distinctive than those for attention to auditory stimuli, and differences among them were statistically significant ( $p<0.01$ between 3D and 2D, and between 3D and Still; $p<0.05$ between 2D and Still). The sum of the amounts of attention directed to the sound and image were 10.0, 10.9, and 11.9 for still image, 2D video, and $3 \mathrm{D}$ video, respectively ( $p>0.05$ for all pairs). This result supports the hypothesis assumed in the probe stimulation method that the total amount of attention is constant. This finding suggested that the attention to visual stimu-

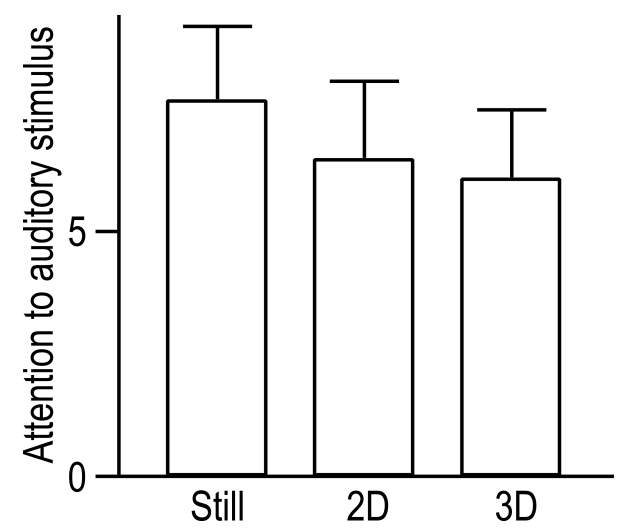

Fig. 1 Subjective evaluation of attention to auditory stimulus while watching the still image (Still), and twodimensional (2D) video, and three-dimensional (3D) video. lus increased owing to the difference in interest among the still image, 2D video and 3D video, while the total amount of attention was constant [10].

\subsubsection{Interestingness of the video}

To examine the effect of the interestingness of the video, a subjective evaluation of the video was conducted for each condition (Fig. 3).

The subjective evaluation of the interest of images was the highest in $3 \mathrm{D}$ video, followed by $2 \mathrm{D}$ videos and still image (in that order), thus exhibiting the same trend as the subjective evaluation of the amount of attention directed toward the image and videos. The differences between still and 2D/3D were statistically significant $(p<0.01)$ although the difference between 2D and 3D was not $(p>0.05)$. This implies that the interestingness

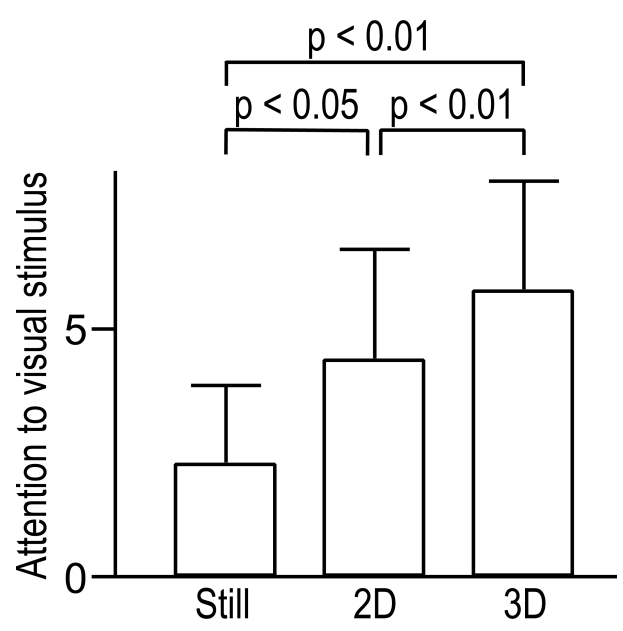

Fig. 2 Subjective evaluation of attention to visual stimulus while watching the still image, $2 \mathrm{D}$ video, and 3D video.

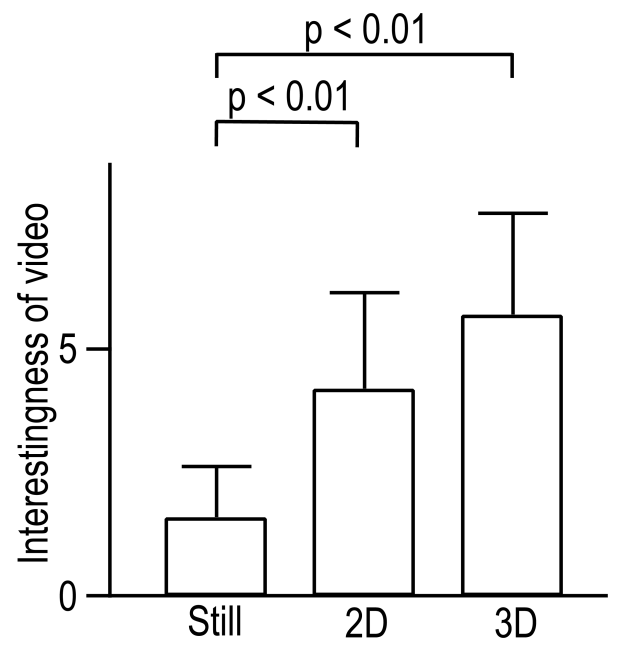

Fig. 3 Subjective evaluation of interestingness of the still image, 2D video, and 3D video. 
of video increased the attention to visual stimulus, as shown in previous study with interestingness adjusted by different numbers of repetition of the same video [10]. The increased interestingness in the present study did not affect the sum of the amount of attention directed to sound and videos, which supports the constant amount of attentional resources assumed in the probe method.

\subsection{Behavioral measures}

\subsubsection{Reaction time}

Subsequently, we examined the extent to which the shift of attention could be confirmed by measuring behavioral measures (Fig. 4).

The reaction time from presentation of the target stimulus to the instant at which the button was pressed was fastest in still image, followed by $3 \mathrm{D}$ video and 2D video (in that order). The differences between all the pairs were not statistically significant $(p>0.05)$ owing to the large SD. This indicates that although the reaction time may be affected by the difference in the image and videos, it is not associated with the differences in the image and videos with different degrees of immersive effects.

\subsubsection{Missed trial rate}

The percentage of missed trials was evaluated (Fig. 5). The missed trial rate increased in the following order: 2D video, still image, and 3D video. However, the difference was within $1.1 \%$, and the SD was large. Thus, all the differences were not statistically significant $(p>0.05$ for all pairs). Moreover, there was no missed trial in eight out of 30 serial trials. This shows that the missed trial rate is not associated with the differences in the image and videos.

\subsubsection{False alarm (FA) rate}

The FA rate was also examined (Fig. 6). The FA in-

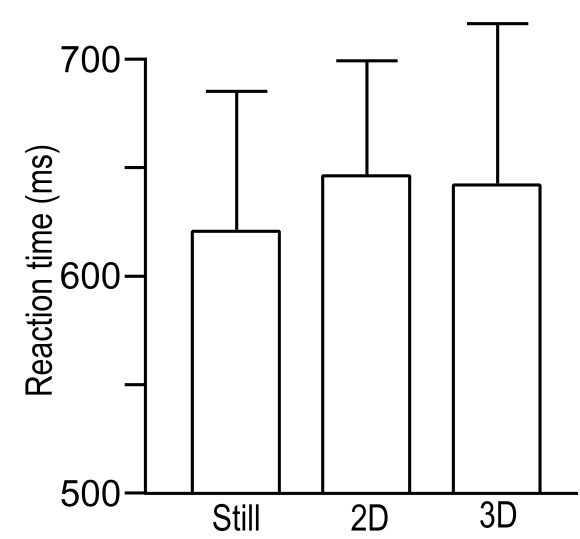

Fig. 4 Reaction time from presentation of the target stimulus for the still image, 2D video, and 3D video. creased in the following order: still image, 2D video, and $3 \mathrm{D}$ video. This finding contradicts with the expected behavior that the FA rate decreases in that order. However, as with the missed trial rate, the overall values were less than $0.21 \%$, and there were no statistically significant differences between all the pairs $(p>0.05)$. Moreover, there was no FA in twenty-two serial trials out of all thirty trials. Therefore, the FA rate is also not associated with the differences in the image and videos.

\subsection{Physiological measures}

The waveforms, peak amplitudes, and latencies of P300 while watching 2D still image, $2 \mathrm{D}$ video, and $3 \mathrm{D}$ video were analyzed, and are shown in Figs. 7-12. P300 was expected to increase in the following order: 3D video, 2D video, and still image. This is attributed to the fact

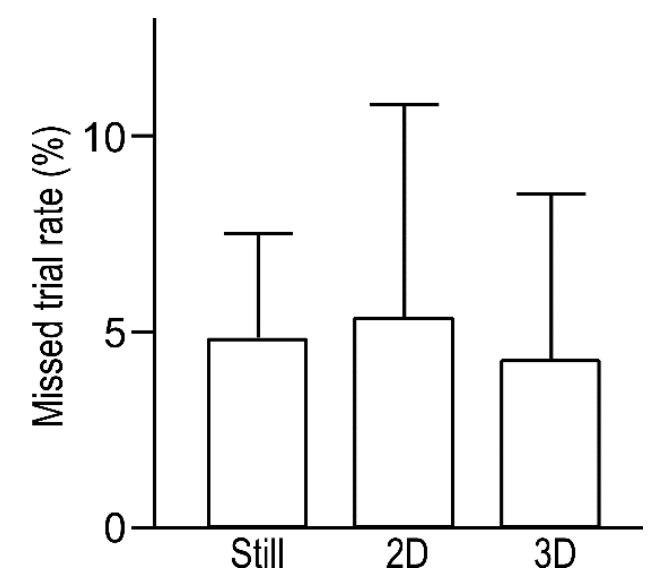

Fig. 5 Missed trial rate defined as no response to the target stimulus, while watching the still image, 2D video, and $3 \mathrm{D}$ video.

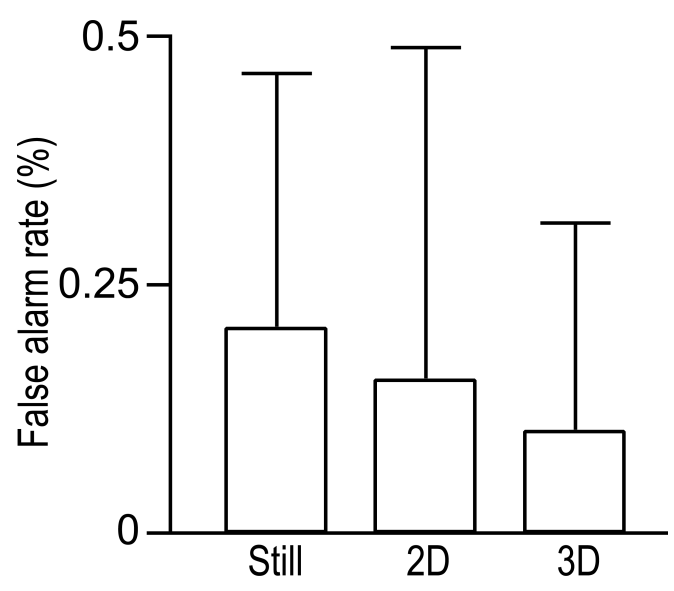

Fig. 6 False alarm rate of incorrectly responded trials among standard and deviant stimulus presentations, while watching the still image, $2 \mathrm{D}$ video, and 3D video. 
that during the probe stimulus method, the more interesting the video is, the lower is the P300 value [10].

\subsubsection{ERP to target stimulus}

Figure 7 shows the averaged waveforms measured at $\mathrm{Pz}$ for the target stimulus $(n=7)$.

The averaged waveform exhibited typical P300 features specific for the target stimulus, with N100, P200, and N200, followed by P300. The amplitude of N100 was not affected by the difference between the image and videos. However, the amplitudes of P300 were smaller in $2 \mathrm{D}$ video than in the others. Next, the latency of P300 was summarized for each condition (Fig. 8).

The peak latencies increased in the following order: still image, 2D video, and 3D video. However, there were no statistically significant differences between all the pairs $(p>0.05)$. Thus, the latency is not associated with the difference in attention to visual stimulus.

Subsequently, we analyzed the peak amplitude of

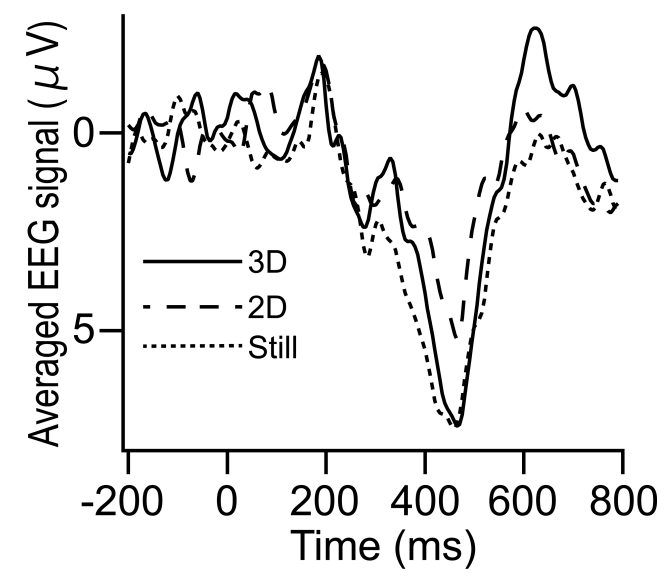

Fig. 7 Averaged event-related potential (ERP) waveforms for target stimuli presented at $0 \mathrm{~ms}$ while watching the $2 \mathrm{D}$ still image, $2 \mathrm{D}$ video, and 3D video.

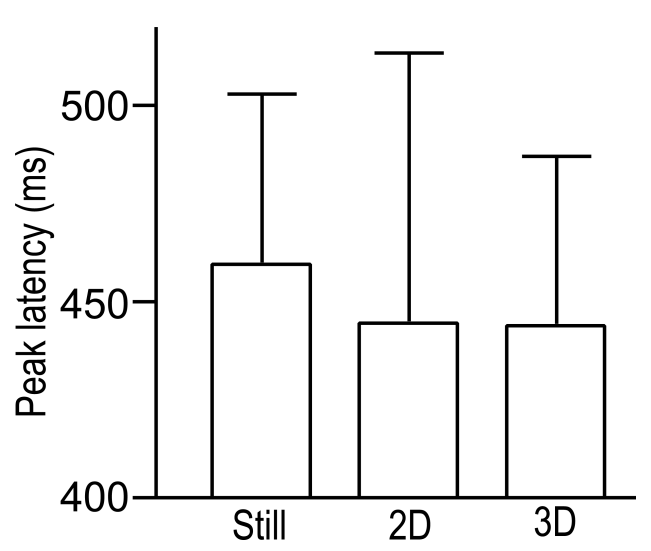

Fig. 8 Latency of P300 for target stimulus while watching the $2 \mathrm{D}$ still image, $2 \mathrm{D}$ video, and 3D video.
P300 (Fig. 9). The peak amplitudes of P300 for the target stimuli increased in the order of still image, 3D video, and 2D video, which were different from the expected result of increase in the order of still image, 2D video, and 3D video. It was difficult to differentiate all the pairs statistically $(p>0.05)$. This was partly because of the large SD for 3D video. Accordingly, the P300 for target stimuli is not associated with the difference in attention to visual stimulus.

\subsubsection{ERP to deviant stimulus}

We then examined P300 for the deviant stimulus, which did not involve the action of pressing the space bar. Figure 10 shows the averaged EEG signals measured at $\mathrm{Cz}$ for the deviant stimulus $(n=10)$.

The averaged waveforms for the deviant stimuli were similar to those for the target stimuli, but there were some major differences. The amplitudes of N100 were larger than those for the target stimuli and were almost equal for the still image and videos. On the contrary,

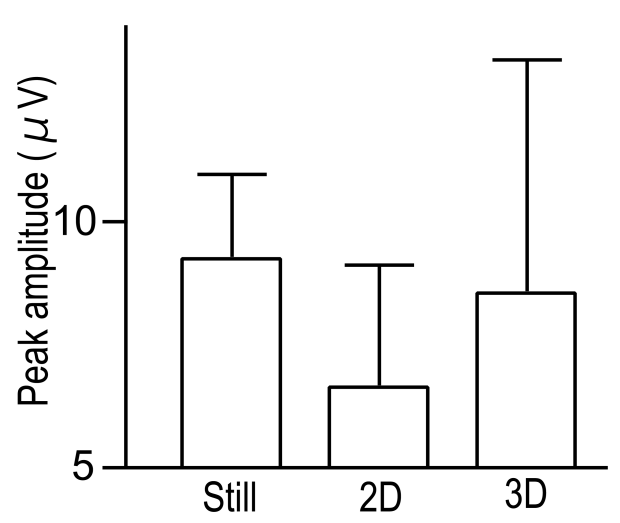

Fig. 9 Amplitude of P300 for the target stimulus while watching the $2 \mathrm{D}$ Still image, $2 \mathrm{D}$ video, and $3 \mathrm{D}$ video.

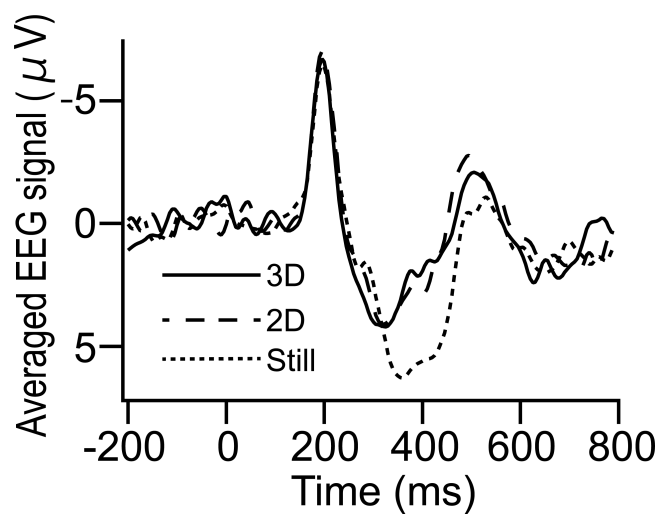

Fig. 10 Averaged ERP waveforms for the deviant stimuli presented at $0 \mathrm{~ms}$ while watching the $2 \mathrm{D}$ still image, $2 \mathrm{D}$ video, and $3 \mathrm{D}$ video. 
P200 and N200 were not observed, as shown for deviant stimulus in previous study [10]. Moreover, the widths of P300 were greater for the target stimuli. Compared to the waveforms for target stimulus, the baseline fluctuation in EEG signal in the order of hundreds ms was greatly reduced for deviant stimulus, so that fewer waveforms needed to be removed. This fluctuation was likely to be caused by the electromyogram from arm and shoulder movements when the participants moved the fingers.

The peak latency for the deviant stimulus was generally smaller than that for the target stimulus (Fig. 11). The peak latency increased in the following order: still image, 2D video, and 3D video for the deviant stimulus. However, all differences were not statistically significant $(p>0.05)$.

The peak amplitude of P300 for the deviant stimulus increased in the following order: still image, 2D video, and 3D video (Fig. 12). The differences were statistical-

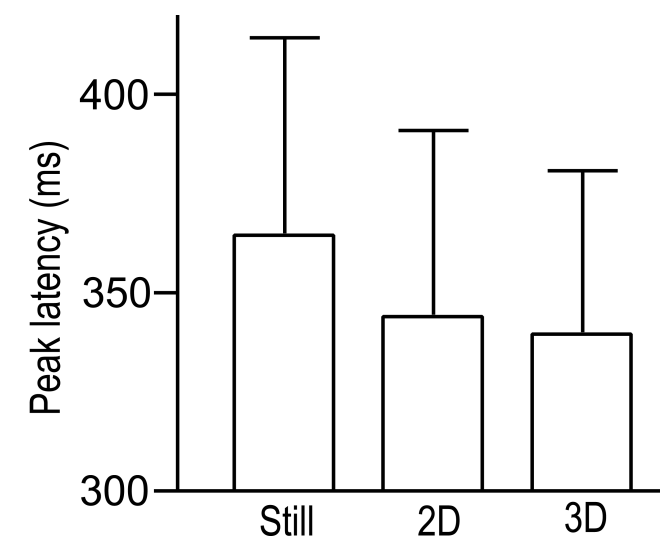

Fig. 11 Latency of P300 for the deviant stimulus while watching the $2 \mathrm{D}$ still image, $2 \mathrm{D}$ video, and $3 \mathrm{D}$ video.

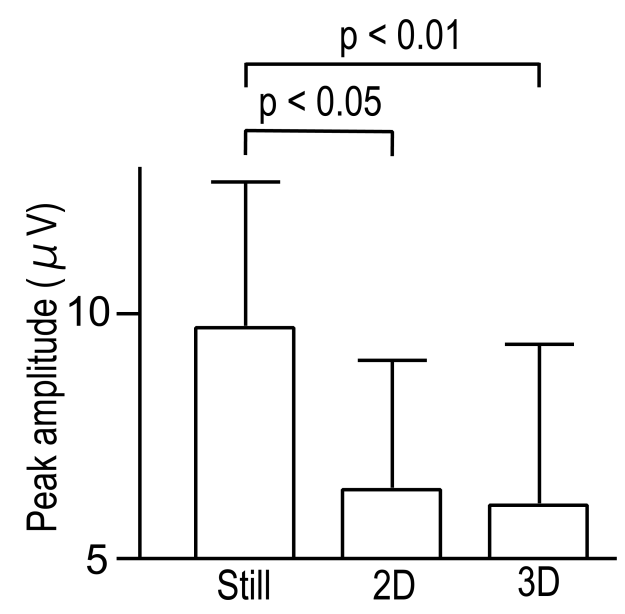

Fig. 12 Amplitude of P300 for the deviant stimulus while watching the $2 \mathrm{D}$ still image, $2 \mathrm{D}$ video, and $3 \mathrm{D}$ video. ly significant between still image and 2D video $(p<$ $0.05)$ and between still image and 3D video $(p<0.01)$, while that between $2 \mathrm{D}$ video and 3D video was not $(p>$ $0.05)$. The differences between the still image and $2 \mathrm{D} / 3 \mathrm{D}$ videos were more statistically significant than those in subjective evaluation of attention to auditory stimulus.

\section{Discussion}

In this study, we investigated whether the attention allocation to still image and 2D and 3D videos can be evaluated by subjective, behavioral, and physiological measures. Firstly, the hypothesis that the attention allocation is more obvious in the order of 3D video, 2D video, and still image was examined by subjective measures. Next, we confirmed that the attention allocation can be evaluated by physiological measures based on the P300 peak amplitude of the deviant stimulus, but not by the behavioral measures.

\subsection{Subjective measures}

In terms of attention to sound, the average rating increased in the order of still image, 2D video, and 3D video, but there were no significant differences. Considering the evaluations among individuals for 2D and 3D videos, we found that three out of ten participants provided higher ratings for $2 \mathrm{D}$ than $3 \mathrm{D}$ video. This suggests that there are significant individual differences. This may be attributed to the randomized order of viewing. Indeed, two out of the five participants that viewed the $2 \mathrm{D}$ video before the $3 \mathrm{D}$ video provided higher ratings for $2 \mathrm{D}$ than $3 \mathrm{D}$ video, while only one out of five other participants that viewed the $3 \mathrm{D}$ video before the $2 \mathrm{D}$ video provided higher ratings for $2 \mathrm{D}$ than $3 \mathrm{D}$ video. Another possible reason may be the fact that calm scenery images were used for the 3D video viewed with HMD to prevent artifacts generated by intense body and viewpoint movements. These factors may contribute to the relatively little difference in effects between 2D and 3D videos.

In terms of attention to image and videos, the average rating increased in the order of $3 \mathrm{D}$ video, $2 \mathrm{D}$ video, and still image. Moreover, the result confirmed that there were statistical differences between all conditions pertaining to the extent of attention directed to the image and videos. Previous studies found that as the attention directed to the video was enhanced, the attention directed to sound became less prominent $[7,8]$. In addition, new videos were rated as more interesting than repeated videos in the subjective measure of video interestingness [10], which could be one of the reasons that videos were rated as more interesting than the still image in the present study.

As expected, the results of the subjective evaluations of interestingness increased in the order of 3D video, 2D 
video, and still image. However, as the sum of the amount of attention directed to the sound and image was not different statistically, the increased interestingness in the present study did not affect the sum of the amount, which supports the constant amount of attentional resources assumed in the probe method.

\subsection{Behavioral measures}

Previous studies have shown that as the attention directed to the image is enhanced, the reaction time to the probe stimulus is increased [11]. This is partly consistent with the results of Shigemitsu et al. [12] in that the best results were obtained in the cases of still images. However, none of the differences pertaining to behavioral measures in the present study was significant. The missed trial rate and FA rate were generally low in the present study. This finding is consistent with the results of Nittono [10], in which no significant differences were found between conditions. Thus, the evaluation of the attention allocation by the behavioral measure was difficult.

\subsection{Physiological measures}

In the ERP waveform for the target stimulus, the peak amplitudes of P300 for still image, 3D video, and 2D video increased in the listed order, and there were no significant differences between all the pairs. Therefore, evaluation with $3 \mathrm{D}$ was difficult for the target stimulus. However, in the ERP waveform for the deviant stimulus, the peak amplitudes of $\mathrm{P} 300$ for $3 \mathrm{D}$ video, 2D video, and still image were increased as expected, and the differences were statistically significant between the still image and $2 \mathrm{D} / 3 \mathrm{D}$ videos.

Regarding the outcomes induced by the ERP waveform in deviant stimulation, our results were in good agreement with those of Nittono [10], both showing that P300 in deviant stimulation appeared with a shorter latency in comparison with the ERP waveform for target stimulation. The P300 wave recorded on the scalp comprises at least two subcomponents; namely, P3a and P3b. The P3a has a shorter latency than P3b by approximately 60-80 ms, and it appears to be dominant at the frontal brain area [4]. This result suggests that $\mathrm{P} 3 \mathrm{a}$ is more abundant than P3b in P300 for the deviant stimulus, and P3b is more abundant than P3a in P300 for the target stimulus. The difference in latency may reflect the different locations, contributions, and roles of P3a and P3b [13, 15]. Furthermore, it can be observed from the P300 amplitude for the deviant stimulus that more attention tended to be allocated to the image in the 3D image condition than in the 2D image condition, which is in good agreement with the proposal of Suzuki et al. [9].

In this study, we evaluated attention allocation in a relatively small sample size $(n=10)$, which is preferable for use in VR content development. However, in some results, the difference between the $2 \mathrm{D}$ and $3 \mathrm{D}$ videos was not clear, or even contradictory to our hypothesis. This may be partly attributed to the small sample size and the lack of difference in interestingness between the two videos, due to the fact that the contents of the 2D and 3D videos were maintained the same to create similar conditions. It is also necessary to use 3D videos with content that is more suitable for VR. In addition, as the order in which the videos were shown was randomized, it is highly possible that the participants became bored after they viewed the first video. Thus, it would be necessary to use new content with strong immersion to obtain more robust results. Furthermore, given that we used auditory probe stimulus in this study, we showed the still image and videos without sound in our experiments. However, given that sound is a very important element for immersion, using somatosensory stimuli as in previous investigations $[12,15]$ would be more effective for future developments.

\section{Conclusion}

In this study, the immersiveness of $2 \mathrm{D}$ and $3 \mathrm{D}$ videos was examined using the probe stimulation method from the viewpoint of attention. We examined whether the amount of attention directed to the VR content can be quantified by the event-related P300 wave response during an oddball task. We confirmed the difference in attention resource allocation by ERP, and consequently showed the possibility of its use for evaluating the sense of immersion. It is expected that the probe stimulation method using ERP will serve as an index for the measurement of the immersiveness of both 2D and 3D videos.

\section{Acknowledgments}

This study was supported by the Chuo University Joint Research Grant and the Chuo University Personal Research Grant.

\section{Conflict of Interest Statement}

The authors declare no conflicts of interest with any companies or commercial organizations per the definition of the Japanese Society for Medical and Biological Engineering.

\section{References}

1. Li L, Yu F, Shi D, Shi J, Tian Z, Yang J, Wang X, Jiang Q: Application of virtual reality technology in clinical medicine. Am J Transl Res. 9(9), 3867-3880, 2017.

2. Jensen L, Konradsen F: A review of the use of virtual reality head-mounted displays in education and training. Educ Inf Technol. 23, 1515-1529, 2018. 
3. Wickens CD, Isreal J, Donchin E: The event related cortical potential as an index of task workload. In A. S. Neal, \& R. F. Palasek (Eds.), Proceedings of the Human Factors Society $21^{\text {st }}$ annual meeting. Santa Monica: Human Factors Society, pp. 282286, 1977.

4. Knight RT, Scabini D. Anatomic bases of event-related potentials and their relationship to novelty detection in humans. J Clin Neurophysiol. 15(1), 3-13, 1998.

5. Comerchero MD, Polich J. P3a and P3b from typical auditory and visual stimuli. Clin Neurophysiol. 110(1), 24-30, 1999.

6. Comerchero MD, Polich J: P3a, perceptual distinctiveness, and stimulus modality. Cogn Brain Res. 7, 41-48, 1998.

7. Comerchero MD, Polich J: P3a and P3b from typical auditory and visual stimuli. Clin Neurophysiol. 110, 24-30, 1999.

8. Katayama J, Polich J: Stimulus context determines P3a and P3b. Psychophysiology. 35, 23-33, 1998.

9. Suzuki J, Nittono H, Hori T: Level of interest in video clips modulates event-related potentials to auditory probes. Int J Psychophysiol. 55, 35-43, 2005.

10. Nittono H: Measuring attention to video clips: An application of the probe stimulus technique using event-related brain potentials. Jpn J Physiol Psychol Psychophysiol. 24(1), 5-18, 2006.

11. Basil, MD: Secondary reaction-time measures. In A. Lang (Ed.), Measuring psychological responses to media messages. Lawrence Erlbaum Associates, Inc., pp. 85-98, 1994.

12. Shigemitsu Y, Hiroshi Nittono H, Hori T: Assessing the allocation of attention to audiovisual experience with the P300 elicited by vibratory probe stimuli. Jpn J Physiol Psychol Psychophysiol. 25(3), 277-285, 2007.

13. Halgren E, Marinkovic K, Chauvel P: Generators of the late cognitive potentials in auditory and visual oddball tasks. Electroencephalogr Clin Neurophysiol. 106(2), 156-164, 1998.

14. Nieuwenhuis S, Aston-Jones G, Cohen JD: Decision making, the P3, and the locus coeruleus-norepinephrine system. Psychol Bull. 131(4), 510-532, 2005.

15. Kida T, Nishihira Y, Hatta A, Wasaka T, Tazoe T, Sakajiri Y, Nakata H, Kaneda T, Kuroiwa K, Akiyama S, Sakamoto M, Kamijo K, Higashiura T: Resource allocation and somatosensory P300 amplitude during dual task: effects of tracking speed and predictability of tracking direction. Clin Neurophysiol. 115(11), 2616-2628, 2004.

\section{Ryo Ogawa}

Ryo Ogawa received his Bachelor of Engineering from Chuo University, Tokyo, Japan in 2021. His research interests include Virtual Reality.

\section{Kaito Kageyama}

Kaito Kageyama is currently an undergraduate student in the Department of Electrical, Electronic, Information and Communication Engineering, Faculty of Science and Engineering, Chuo University. His research interests include Virtual Reality.

\section{Yasushi NAKATANI}

Yasushi NAKaTANi is currently an Associate Professor of the Faculty of Economics at Chuo University. He received the Doctor of Medical Science from Toho University, Tokyo, Japan, in 2008. He is doing research in the field of health science.

\section{Yumie ONo}

Yumie ONo received her $\mathrm{PhD}$ in Electrical Engineering in 2004 from the Waseda University. After she worked as an Assistant and Associate Professor in Waseda University and Kanagawa Dental College, she is currently a Professor in the Department of Electronics and Bioinformatics, School of Sci-

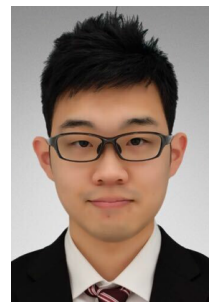
ence and Technology at Meiji University. Her work focuses on the application of noninvasive brain imaging and biomedical measurement techniques on medicine and rehabilitation. She is a member of Japanese Society for Medical and Biological Engineering and the Japan Neuroscience Society, and a councilor of Japan Society of Neurovegetative Research and Japan Biomagnetism and Bioelectromagnetics society. She is also an Associate Editor of the IEEE Transactions on Neural Systems and Rehabilitation Engineering.

\section{Shingo Murakami}

Shingo Murakami received his $\mathrm{PhD}$ in Electrical Engineering in 2001 from the University of Tokyo. After working as an Assistant Professor in New Mexico University, Osaka University and Toho University, he is currently a Professor in the Department of Electrical, Electronic, Information and

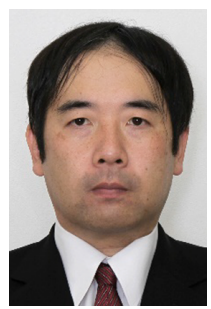
Communication Engineering, Faculty of Science and Engineering, Chuo University. He is a Councilor of the Physiological Society of Japan and the Japanese Pharmacological Society. His research interests cover EEG, permittivity measurements of biological tissues, and photoacoustic imaging. 